\title{
Heralded single-photon states generated via spontaneous parametric down-conversion with an orbital angular momentum controlled by the pump field
}

\author{
D.A. Turaykhanov, D.O. Akat'ev, A.V. Shkalikov, V.S. Romanov, and A.A. Kalachev \\ FRC Kazan Scientific Center, Russian Academy of Sciences, 420029 Kazan, Russia
}

\begin{abstract}
Conditional preparation of single-photon states in the process of spontaneous parametric down-conversion with an orbital angular momentum controlled by the pump field is studied.
\end{abstract}

Currently, much attention is paid to the study and use of light beams with orbital angular

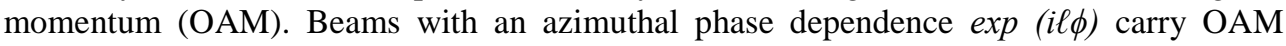
equal to $\ell \hbar$ per photon, where the azimuthal index $\ell$ can take any integer value [1]. Since OAM is theoretically unlimited, this gives access to a lot of states, which is of great interest for classical [2] and quantum [3] communication, studying quantum entanglement [4], and also for quantum teleportation [5]. Today, there are a number of works devoted to the preparation of multidimensional photonic states encoded in different degrees of freedom. As an example, we can mention the work [6], wherein eighteen qubits were entangled using three degrees of freedom - polarization, spatial arrangement, and orbital angular momentum - of only six photons.

The most effective method for synthesizing light beams with OAM is to transform the spatial structure of the beams using a spatial light modulator (SLM). SLM is a liquid crystal screen, each pixel of which can introduce its own phase shift so that almost any phase profile can be set. To generate beams with an orbital angular momentum, we can take advantage of the method developed in [7], which requires only a phase SLM, while for measuring OAM, a compensation method developed in [9] can be used.

One of the simplest methods for generating single photons is the process of spontaneous parametric down-conversion (SPDC) of light, during which the photons of a pump beam incident on a nonlinear crystal are annihilated with spontaneous creation of two photons, called idler and signal. This process satisfies the conditions of phase matching in the time and space domains, which are usually written as energy and momentum conservation laws, respectively: $\omega_{p}=\omega_{i}+\omega_{s}$ and $\overrightarrow{\mathrm{k}}_{p}=\overrightarrow{\mathrm{k}}_{i}+\overrightarrow{\mathrm{k}}_{s}$. Here $\omega_{n}$ and $\overrightarrow{\mathrm{k}}_{n}$ are the frequency and wave vector of the signal photon $(n=s)$, idler photon $(n=i)$ and pump photon $(n=p)$. Another distinctive feature of the SPDC process is the conservation law of OAM, which was demonstrated in $[9,10]$. This property provides a wide range of possibilities for the 
preparation of single-photon states via SPDC. For collinear SPDC, the following condition is true [8]: $\ell_{p}=\ell_{s}+\ell_{i}$, where $\ell_{n}$ is the value of OAM of $n$th photon $(n=\{p, s, i\})$.

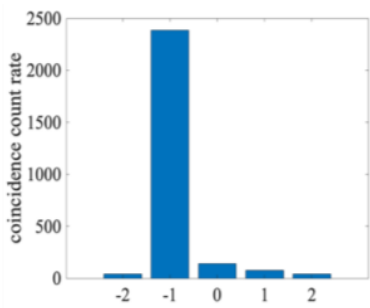

(a)

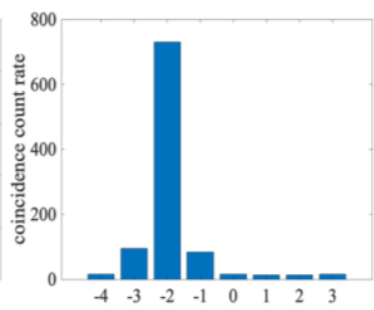

(b)

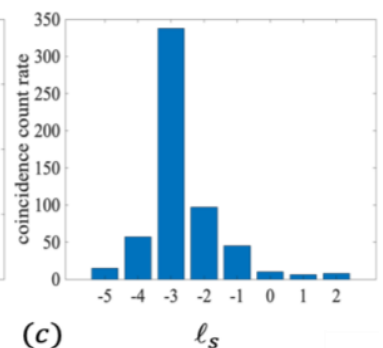

(c)

Fig. 1. Coincidence count rates as a function of detection modes azimuthal number $\ell_{S}$. The cases of the pump beam with a) $\ell_{p}=1$, b) $\ell_{p}=2$, c) $\ell_{p}=3$

In the present work, we study the process of collinear SPDC in PPLN crystal providing generation of photon pairs at the wavelengths of $810 \mathrm{~nm}$ and $1550 \mathrm{~nm}$ pumping by the second harmonic of a cw Nd laser. Pump laser is filtered using a single-mode $(500 \mathrm{~nm})$ fiber, and then sent to the right side of the SLM, where light beam reflects with OAM. Next, the pump beam focuses into a nonlinear crystal, when corelated photon pairs at 810 and $1550 \mathrm{~nm}$ is generated via SPDC process.

In one channel called "idler", photons are coupled into single-mode fiber. In this case, detected photons have only zero OAM $\left(\ell_{i}=0\right)$. In another channel called "signal", the left side of the SLM modulator was used, where the phase masks is changed to measurements the OAM value of the photons. After reflection from SLM, the signal beam was injected into a single-mode fiber, and as a result, maximum coincidence was obtained when compensating photons have OAM with gaussian mode.

From the experimental conditions described above, when a single photon with only zero OAM is detected, the orbital angular momentum of the signal photon corresponds to the orbital angular momentum of pump. In the figure 1 it can be seen that the coincidence counting rate is maximum when the detecting mask is inverse to the OAM of pump.

Financial support from the government assignment for FRC Kazan Scientific Center of RAS

\section{References}

1. L. Allen, M. W. Beijersbergen et al. // Phys. Rev. 1992. A 45, 8185-8189

2. G. Gibson, J. Courtial et al. // Opt. Express. 2004. 12, 5448-56

3. M. Mirhosseini, O.S. Magana-Loaiza et al. // New J. Phys. 2015. 17, 033033

4. A. Vaziri, A. WeihsGand Zeilinger et al. // Phys. Rev. Lett. 2002. 89, 240401

5. A.Z. Khoury, P. Milman // Phys. Rev. A. 2011. vol. 83, no. 6, p. 060301

6. Xi-Lin Wang, Yi-Han Luo, at all. // Phys. Rev. Lett. 2018. 120, 260502

7. Bolduc E., Bent N., Santamato E. et al. // Optics letters. 2013. Vol. 38, no. 18. P. 35463549.

8. Mair A. et al. // Nature. 2001. 412, 313. 